\title{
Amnioinfusión transcervical en trabajo de parto con meconio espeso
}

\author{
Claudia Parra ${ }^{*}$; Arturo Cuevas*; Christian Pérez**, Pedro Rey**
}

\begin{abstract}
RESUMEN: El objetivo de este estudio es evaluar el efecto de la amnioinfusión salina transcervical sobre el resultado perinatal en pacientes con trabajo de parto complicado con meconio.

Estudio prospectivo con control histórico, realizado en el Hospital Ramón González Valencia, en 115 pacientes en trabajo de parto con meconio espeso, de las cuales 55 fueron sometidas a amnioinfusión.

EI volumen de solución salina oscila entre 500 y $800 \mathrm{cc}$. En el grupo de amnioinfusión hubo disminución en la frecuencia de puntajes bajos de Apgar (<7), reducción en el hallazgo de meconio debajo de cuerdas vocales $(12,7 \%$ Vs. 50,9\%) y en la necesidad de ventilación mecánica positiva $(1,9 \%$ Vs. $33,3 \%)$.

Concluímos que la amnioinfusión transcervical mejora el resultado perinatal reduciendo la incidencia de síndrome de Broncoaspiración de meconio, disminuye el síndrome de dificultad respiratoria neonatal, presencia de meconio bajo cuerdas vocales y necesidad de ventilación mecánica.
\end{abstract}

PALABRAS CLAVES: Amnioinfusión, síndrome de broncoaspiración de meconio.

SUMMARY: Our purpose was to evaluate the effect of transcervical amnioinfusion in the management of labor complicated by meconium.

This prospective study with historic control group was performed at hospital Ramón González Valencia. Fifty five Iaboring pregnant women with particulate meconium were recived amnioinfusion.

The average of infusion saline was 500-800 c.c significant diferences between the amnioinfusion group and the control group were found for the following: Decreased frecuence of lower Apgar scores $(<7)$ at amnioinfusion group.

Meconium below the vocal cords was reduced in the study group (12,7\% Vs. 50,9\%) and the need for positive pressure ventilation (1,9\% Vs. 33,3\%).

We conclude that transcervical amnioinfusion improved neonatal outcome by reduction in the incidence of meconium aspiration syndrome, decreases the frecuence of meconium below the vocal cords and the need for positive pressure ventilation.

KEY WORDS: Amnioinfusion, meconium aspiration syndrome.

\section{Introducción}

El síndrome de Broncoaspiración de meconio (SBAM) es causado posiblemente por obstrucción mecánica y neumonitis química debido a la aspiración de meconio espeso en tráquea y pulmones fetales (1).

La incidencia de líquido amniótico meconiado (LAM) es $8-17 \%$ de todos los partos siendo $46 \%$ meconio espeso (2).

El síndrome de broncoaspiración de meconio (SBAM) ocurre en $1-3 \%$ de todos los casos de LAM y en $10-30 \%$ de recién nacidos con meconio bajo cuerdas vocales (3).

\footnotetext{
1 Departamento de Ginecología y Obstetricia. Universidad Industrial de Santander.

* Especialistas en Ginecología y Obstetricia.

** Profesores. Departamento de Ginecología y Obstetricia. Bucaramanga, Colombia.
}

En 1983 Miyasaki y Taylor (12) describieron el uso de amnioinfusión intraparto para el manejo de desaceleraciones variables. Posteriormente, Westrom y Parsons (9) evalúan la eficacia de la amnioinfusión para prevenir aspiración de meconio.

El objetivo del presente estudio es evaluar el efecto de la amnioinfusión sobre el resultado perinatal en pacientes con trabajo de parto complicado con meconio espeso; a su vez de terminar el impacto sobre la vía de terminación del embarazo, frecuencia de SBAM, variación de ingresos a Unidad de Cuidados Intensivos Pediátrico y medir el efecto del procedimiento sobre la mortalidad fetal.

\section{Materiales y métodos}

El estudio fue realizado en el Hospital Ramón González Valencia en el Departamento de Ginecología y Obstetricia de la Universidad Industrial de Santander en el período comprendido entre abril 94 y abril 95 . 
Los criterios de inclusión fueron: Edad gestacional mayor o igual a 37 semanas, pacientes en trabajo de parto con membranas rotas (espontánea o artificial), líquido amniótico meconiado espeso y embarazo único en presentación de vértice.

Criterios de Exclusión: Sufrimiento fetal agudo no recuperable in útero (monitoreo omnioso), embarazo múltiple, sangrado genital, malformaciones fetales mayores, coriamnionitis y presentaciones diferentes al vértice.

A todas las pacientes se les realizó consentimiento por escrito.

El grupo control se seleccionó retrospectivamente; de las historias clínicas con diagnóstico de LAM espeso, eligiendo aleatoriamente 60 durante el período 93-94. Estas pacientes recibieron manejo convencional. En este grupo se determinaron las variables: vía de terminación del parto, Apgar al minuto, 5 y 10 minutos, presencia de meconio bajo cuerdas vocales, puntaje de Silveman Anderson, ingreso a la Unidad de Cuidados Intensivos pediátrico, necesidad de ventilación mecánica, muerte o no. Todas las pacientes tuvieron monitoreo continuo externo.

Las pacientes en el grupo estudio, recibieron amnioinfusión transcervical con solución salina normal a $37^{\circ} \mathrm{C}$ con catéter conectado a equipo de infusión a una infusión de flujo de $16 \mathrm{cc} / \mathrm{min}$ (500 $\mathrm{cc}$ en 30 minutos), la infusión posterior fue de $150 \mathrm{ml} / \mathrm{hora}$.

Todos los recién nacidos recibieron manejo obstétrico pediátrico que incluyó aspiración endotraqueal según lo descrito por Carson (7-8).

El estudio incluyo 115 pacientes, 55 pacientes en el grupo de amnioinfusión y 60 en el grupo de control.

\section{Resultados}

La información obtenida fue introducida en una base de datos utilizando el programa estadístico Epi-Info 5.1.

Se considera diferencia significativa cuando la probabilidad (p) es inferior al 5\% (0.05). La tabla 1 compara las características demográficas de los dos grupos. No hubo diferencia significativa.

Ante el mínimo número de pacientes con Apgar inferior a 4 (hipoxia severa) se analiza la presencia de hipoxia como resultado perinatal negativo como punto de corte Apgar $\leq 6$.

El Apgar fue medido al minuto, 5 y 10 minutos siendo significativamente diferente entre los grupos (gráficas 1 y 2). En ellas se observa que las pacientes del grupo de control tienen más probabilidad de sufrir hipoxia evaluada por Apgar inferior a 7 que aquellas sometidas al procedimiento; siendo esta probabilidad 3,36 veces al minuto; 2,37 a los 5 minutos y 1,7 a los 10 minutos. Se consideró como dificultad respiratoria un Silveman Anderson superior a tres. Las diferencias son significativas, en las pacientes del grupo control hay 2,32 veces más probabilidad de presentar dificultad respiratoria en el recién nacido que en las del grupo de estudio.

El $50.9 \%$ de las pacientes del grupo control presentaron meconio bajo cuerdas vocales en comparación con el $12.7 \%$ del grupo de amnioinfusión (tabla 3 ).
Tabla 1

CARACTERISTICAS DE LAS PACIENTES

\begin{tabular}{|llll|}
\hline & $\begin{array}{l}\text { Amnioinfusión } \\
\mathbf{N}=\mathbf{5 5}\end{array}$ & $\begin{array}{l}\text { Control } \\
\mathbf{N}=\mathbf{6 0}\end{array}$ & Significancia \\
\hline Edad materna & 24.62 & 24.68 & $\mathrm{Ns}$ \\
$\begin{array}{l}\text { Primigestantes } \\
\text { Nulíparas }\end{array}$ & $6(10.9 \%)$ & $12(20 \%)$ & $\mathrm{Ns}$ \\
$\begin{array}{l}\text { Edad } \\
\text { gestacional }\end{array}$ & 40.14 & $29(48.3 \%)$ & $\mathrm{Ns}$ \\
Peso fetal & $3389+459 \mathrm{gr}$ & $3335+459 \mathrm{gr}$ & $\mathrm{Ns}$ \\
\hline
\end{tabular}

Ns: No significativa

Gráfica 1

APGAR AL MINUTO

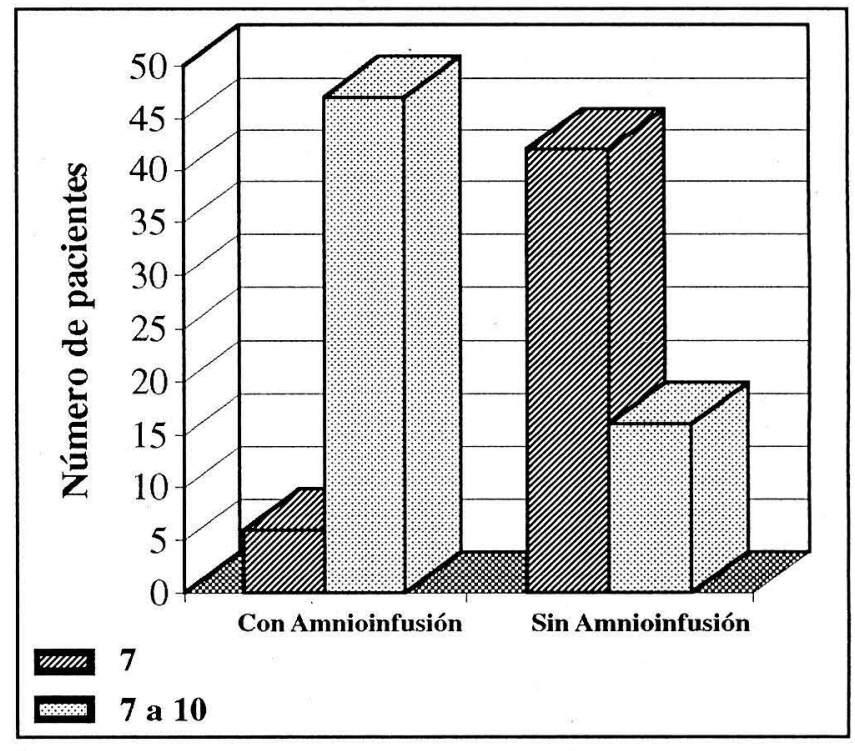

Gráfica 2

APGAR A LOS CINCO MINUTOS

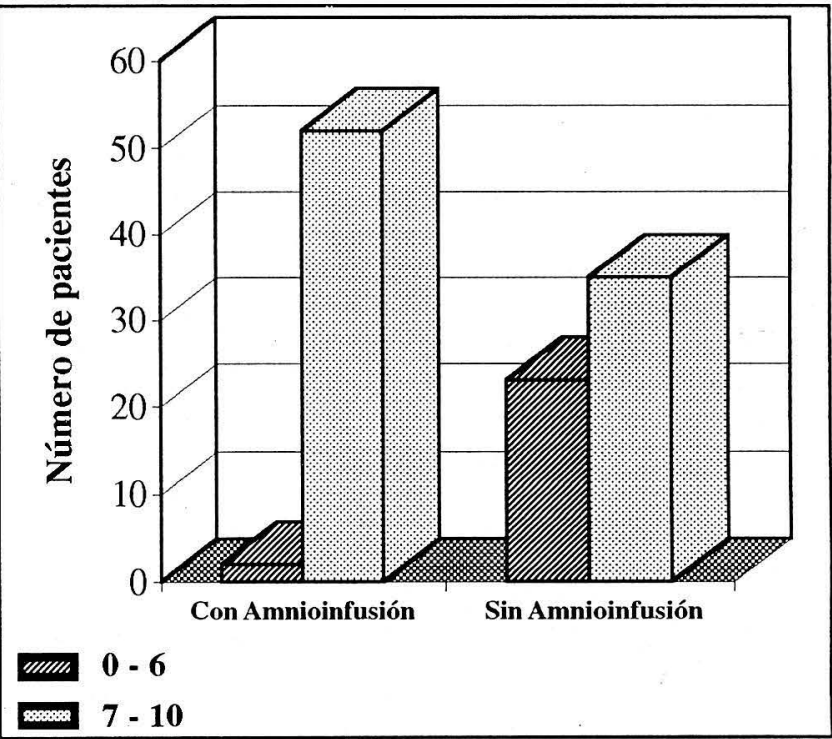


Tabla 2

FORMA DE TERMINACION DEL PARTO

\begin{tabular}{|lll|}
\hline & Amnioinfusión & Control \\
\hline Cesárea & $23(41.68 \%)$ & $22(36 \%)$ \\
Vaginal & $32(58.2 \%)$ & $38(63.3 \%)$ \\
Total & 55 & 60 \\
\hline
\end{tabular}

RR 0.88 (IC 0.5\%: $056-1.38$ )

$\mathrm{x} 2=0.32-1 \mathrm{gl}-\mathrm{P}<0.573$

Tabla 3

PRESENCIA DE MECONIO DEBAJO DE CUERDAS VOCALES

\begin{tabular}{|l|c|c|c|}
\hline \multirow{2}{*}{ Grupo } & \multicolumn{2}{|c|}{ Bajo cuerdas vocales } & \multirow{2}{*}{ Total } \\
\cline { 2 - 3 } & $\begin{array}{c}\text { Meconio } \\
+\end{array}$ & $\begin{array}{c}\text { Meconio } \\
-\end{array}$ & \\
\hline Control & $29(50.9 \%)$ & $28(49.1 \%)$ & 57 \\
Amnioinfusión & $7(12.7 \%)$ & $48(87.3 \%)$ & 55 \\
Total & 36 & 76 & 112 \\
\hline
\end{tabular}

RR 2.19 (IC 95\%: 1.56) 3.06

$\mathrm{X} 2=18.51-1 \mathrm{gl}-\mathrm{P}<0.0000017$

Tabla 4

NECESIDAD DE VENTILACION MECANICA

\begin{tabular}{|c|c|c|}
\hline \multirow[t]{2}{*}{ Grupo } & \multicolumn{2}{|c|}{ Ventilación mecánica } \\
\hline & + & - \\
\hline Control & $20(33.3 \%)$ & $408(66.3 \%)$ \\
\hline Amnioinfusión & $1(1.9 \%)$ & $53(98.1 \%)$ \\
\hline Total & 21 & 73 \\
\hline
\end{tabular}

$\mathrm{RR}=18 \mathrm{IC} 95 \% 2.5-129.7$

$\mathrm{X} 2=18.58-1 \mathrm{gl}-\mathrm{p}<0.000163$

En el grupo estudio el $16.7 \%$ de las pacientes requirió Unidad de Cuidados Intensivos, en el grupo control el $35 \%$. Con respecto a la necesidad de ventilación mecánica hay diferencia significativa entre los grupos, el control tiene 18 veces más necesidad de ventilación mecánica que el grupo amnioinfusión (tabla 4).

No hubo muertes en el grupo amnioinfusión: la mortalidad del grupo control es de $16.7 \%$. El volumen de infusión de solución salina normal osciló entre 500 y $800 \mathrm{cc}$.

Se observaron complicaciones menores en 14 pacientes (25.5\% del grupo amnioinfusión), de los cuales sólo el $3.6 \%$ obligó a suspender el procedimiento. Las más frecuentes fueron dolor en el segmento uterino $(35.7 \%)$, dificultad en la introducción del catéter (21.43\%) y laceración cervical por el procedimiento (14.29\%).

\section{Discusión}

La amnioinfusión como manejo del trabajo de parto complicado con LAME fue utilizado por primera vez en 1969 por Sadovski (2) reportando mejoría del resultado perinatal, que ha sido confirmado por otros autores $(3,9-10)$ con trabajos que incluyeron entre 40 y 170 pacientes siendo en su totalidad prospectivos aleatorios.

Los diferentes estudios plantean criterios de inclusión y exclusión que difieren de los nuestros.

1. Wenstrom (9) incluyó sólo pacientes con meconio espeso, excluyendo barro meconial y alteraciones al monitoreo que indicaran sufrimiento fetal. Nuestro estudio sólo excluyó monitoreos ominosos.

2. Sadovski (2) incluyó gestaciones $>34$ semanas a diferencia del nuestro con edad gestacional $>37$ semanas

3. Macri (10) incluyó oligohidramnios, presentaciones de no vértex y patrones de fetocardia normal con dilataciones cervicales $\leq 5$. Diferimos en los patrones de monitoreo y en la realización del procedimiento en cualquier estado de dilatación cervical.

4. Cialone (3) excluyó cualquier condición obstétrica además de la presencia de meconio en el LA.

Encontramos disminución en la frecuencia de puntajes bajos de Apgar $(<7)$ a los minutos 1,5 y 10 en productos sometidos a la amnioinfusión comparada con el grupo control en un $11.1 \%$ Vs. $71.7 \%$ al minuto; $1.92 \%$ Vs. $40 \%$ a los 5 minutos, $0 \%$ Vs. $64.9 \%$ a los 10 minutos.

Los estudios internacionales (11) evalúan el estado ácido-básico neonatal con gasimetría en sangre del cordón siendo este parámetro más preciso en la evaluación del resultado perinatal.

Teniendo como probable indicador de Síndrome de Broncoaspiración de Meconio el ingreso a UCI por Síndrome de Dificultad Respiratoria severa observamos su notable disminución en el grupo amnioinfusión.

Hubo disminución en la frecuencia de meconio bajo cuerdas vocales en el grupo de estudio comparadas con el grupo control.

En la literatura hay una disminución en la incidencia de SBAM con amnioinfusión de un $80 \%$ (11). No encontramos diferencia significativa en la vía de terminación del embarazo en ambos grupos en contraposición con los otros estudios en los que la amnioinfusión disminuye la frecuencia de intervención cesárea.

Se considera lógica la realización de esta técnica en el manejo de trabajo de parto complicado con LAME por dos razones:

La amnioinfusión corrige el oligohidramnios y puede diluir el meconio espeso de tal forma que disminuye los efectos tóxicos de la aspiración; y corrige el estímulo vagal secundario a la compresión del cordón umbilical.

\section{Conclusiones}

1. La amnioinfusión transcervical con solución salina normal mejora el resultado perinatal reduciendo la incidencia de SBAM.

2. La amnioinfusión es efectiva en la dilución del meconio. 
3. Disminuye el Síndrome de Dificultad Respiratoria neonatal, la presencia de meconio bajo cuerdas vocales y la necesidad de ventilación mecánica.

4. La proporción de recién nacidos con Apgar menor de 7 al minuto fue significativamente menor en el grupo amnioinfusión.
5. No hubo diferencia en la vía de terminación del embarazo.

6. Es un procedimiento efectivo, seguro y de fácil realización.

\section{BIBLIOGRAFIA}

1. Jwendel Paul. The current State of Amnioinfusion Postgraduate Obstetrics Gynecology 1996; 16(14).

2. Sadovski Yoel. Prophylactic amnioinfusion during labor complicated by meconium: A preliminar report. Am. J. Obstet. Gynecol. 1989; 161: 61 3-17.

3. Cialone Paw et al. Amnioinfusion during labor complicated by particulate meconium-stained fluid decreases neonatal morbidity. Am. J. Obstet. Gynecol. 1994; 170: 842-849.

4. Nathan Lawrence. Correlation of measured amniotic fluid volume and sonographic predictors of oligohidramnios. Obstet. Gynecol. $1994 ; 83$.

5. Berkus Michael et al. Meconium stained Amniotic fluid: Increased Risk for adverse Neonatal outcome Obstet. Gynecol. 1994; 84: 115-120.

6. Nathan Lawrence et al. Meconium: A 1990's Perspective on an old Obstetric Hazard Obstetric Gynecol 1994; 83: 329-332.
7. Carson BS Lose y RW Bowe. Combined Obstetric and pediatric approach to prevent meconium aspiration sindrome. Am. J. Obstet. Gynecol. 1976; 126: 712-715.

8. Bradley A Yoder. Meconium Stained Amniotic fluid and respiratory complications: Impact of selective traqueal suction. Obstetric Gynecol 83: 77-84.

9. Westrom DK., Parsons MT. The prevention of meconium aspiration in labor using amnioinfusion Obstet. Gynecol. 1989; 73: 647-652.

10. Macri CJ., Schimmer DB., Leung A Greenspoon JS. Amnioinfusion improve the outcome of labor complicated by meconium aspiration sindrome. Am. J. Obstet. Gynecol. 1992; 166: 405.

11. Dye Timothy Aubry R. Grois S. Amnioinfusion and the intrauterine prevention of meconium aspiration. A metanalysis. Am. J. Obstet. Gynecol. 1994; 171: 160-165.

12. Miyasaki FS., Taylor NA. Saline Amnioinfusion for relief of variable desacelerations: a prospective randomized study. Am. J. Obstet. Gynecol. 1983; 146: 670-678.

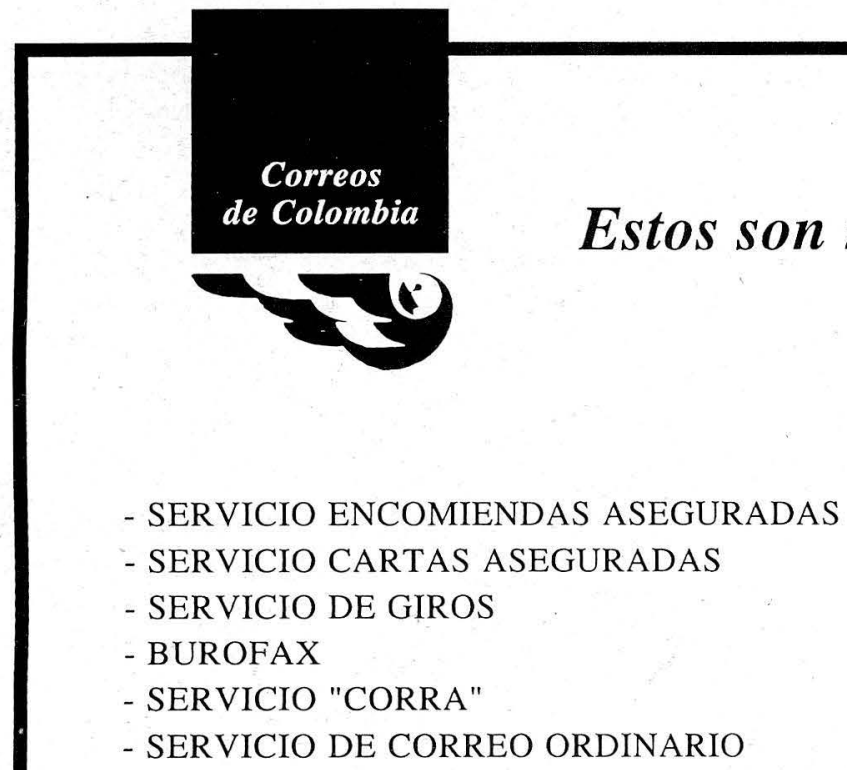

\footnotetext{
- SERVICIO DE CORREO CERTIFICADO

- SERVICIO DE CERTIFICADO ESPECIAL

- ENCOMIENDAS CONTRA REEMBOLSO

- SERVICIO TARIFA POSTAL REDUCIDA

- SERVICIO DE FILATELIA

- SERVICIO ELECTRONICO

- SERVICIO INTERNACIONAL APR/SAL

- SERVICIO RESPUESTA COMERCIAL

- SERVICIOS ESPECIALES
}

Teléfonos para quejas y reclamos: 3340304 - 3415536 - Santafé de Bogotá, D.C. - Colombia Cuente con nosotros hay que creer en los Correos de Colombia 